Supporting Information

\title{
Silole-Containing Polymer Nanodot: An Aqueous Low-Potential Electrochemiluminescence Emitter for Biosensing
}

Yaqiang Feng, ${ }^{\dagger, \S}$ Chunhui Dai, ${ }^{\ddagger, \S}$ Jianping Lei, ${ }^{\dagger}$ Huangxian $\mathrm{Ju}^{,{ }^{*, \dagger}}$ and Yixiang Cheng ${ }^{\star, \ddagger}$

† State Key Laboratory of Analytical Chemistry for Life Science, School of Chemistry and Chemical Engineering, Nanjing University, Nanjing 210023, P. R. China

\# Key Lab of Mesoscopic Chemistry of MOE, School of Chemistry and Chemical Engineering, Nanjing University, Nanjing 210023, P. R. China

${ }^{*}$ Corresponding author. Phone/Fax: $+86-25-83593593$

E-mail: hxju@nju.edu.cn,yxcheng@nju.edu.cn 


\section{Synthesis of Silole-Containing Polymer (SCP)}

M1, 3,7-dibromo-2,8-dimethoxy-5,5-dioctyl-5H-dibenzo[b, $d]$ silole, and M2, 3,6-diethynyl9-octyl-9H-carbazole were prepared according to the reported procedures. ${ }^{1,2}$ As shown in Scheme $\mathrm{S} 1$, a mixture of M1 (156.14 g, $0.25 \mathrm{mmol}), \mathbf{M} 2(81.75 \mathrm{mg}, 0.25 \mathrm{mmol}), \mathrm{Pd}\left(\mathrm{PPh}_{3}\right)_{4}(8.7 \mathrm{mg}, 0.0075$ $\mathrm{mmol})$ and $\mathrm{CuI}(1.43 \mathrm{mg}, 0.0075 \mathrm{mmol})$ was added in Schlenk tube. Dry $\mathrm{Et}_{3} \mathrm{~N}(20 \mathrm{~mL})$ was added by syringe after the tube was evacuated under vacuum and refilled with $\mathrm{N}_{2}$ for three times. The mixture was stirred at $80{ }^{\circ} \mathrm{C}$ for $24 \mathrm{~h}$ under $\mathrm{N}_{2}$ atmosphere. The resulting mixture was cooled to room temperature. After the resulting ammonium salt was filtered off and the filtrate was concentrated under reduced pressure, the residue was dissolved in a small quantity of THF. The resulting solution was then added into $50 \mathrm{~mL}$ of methanol to precipitate the polymer, which was dried in a vacuum to give $104.97 \mathrm{mg}$ of a yellow solid in a 53\% yield. $M_{w}=8706, M_{n}=6107$, $\mathrm{PDI}=1.43 .{ }^{1} \mathrm{H}$ NMR $\left(300 \mathrm{MHz}, \mathrm{CDCl}_{3}\right): \delta 8.33(\mathrm{~s}, \mathrm{Ar}-\mathrm{H}), 7.76-7.69$ (m, Ar-H), 7.39-7.31 (m, Ar-H), 4.33-4.28 (t, $\left.\mathrm{OCH}_{3}\right), 4.12-4.03\left(\mathrm{~m}, \mathrm{NCH}_{2}\right), 1.89\left(\mathrm{~s}, \mathrm{NCH}_{2} \mathrm{CH}_{2}\right), 1.64\left(\mathrm{~s}, \mathrm{NCH}_{2} \mathrm{CH}_{2}\right), 1.25-1.21(\mathrm{~d}$, $\left.\mathrm{CH}_{2} \mathrm{CH}_{2} \mathrm{CH}_{2}\right), 0.88-0.83\left(\mathrm{~m}, \mathrm{CH}_{3}\right)$.

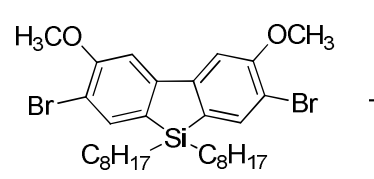

M1

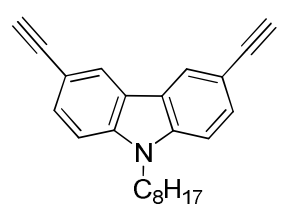

M2

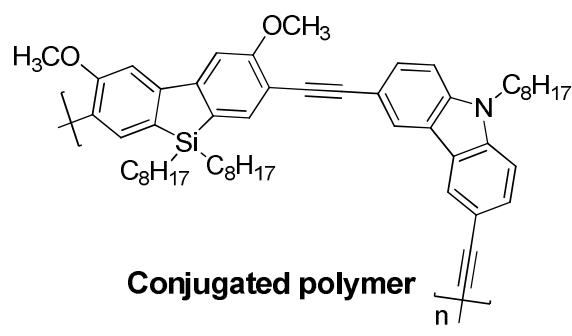

Scheme S1. Synthetic route of SCP. 


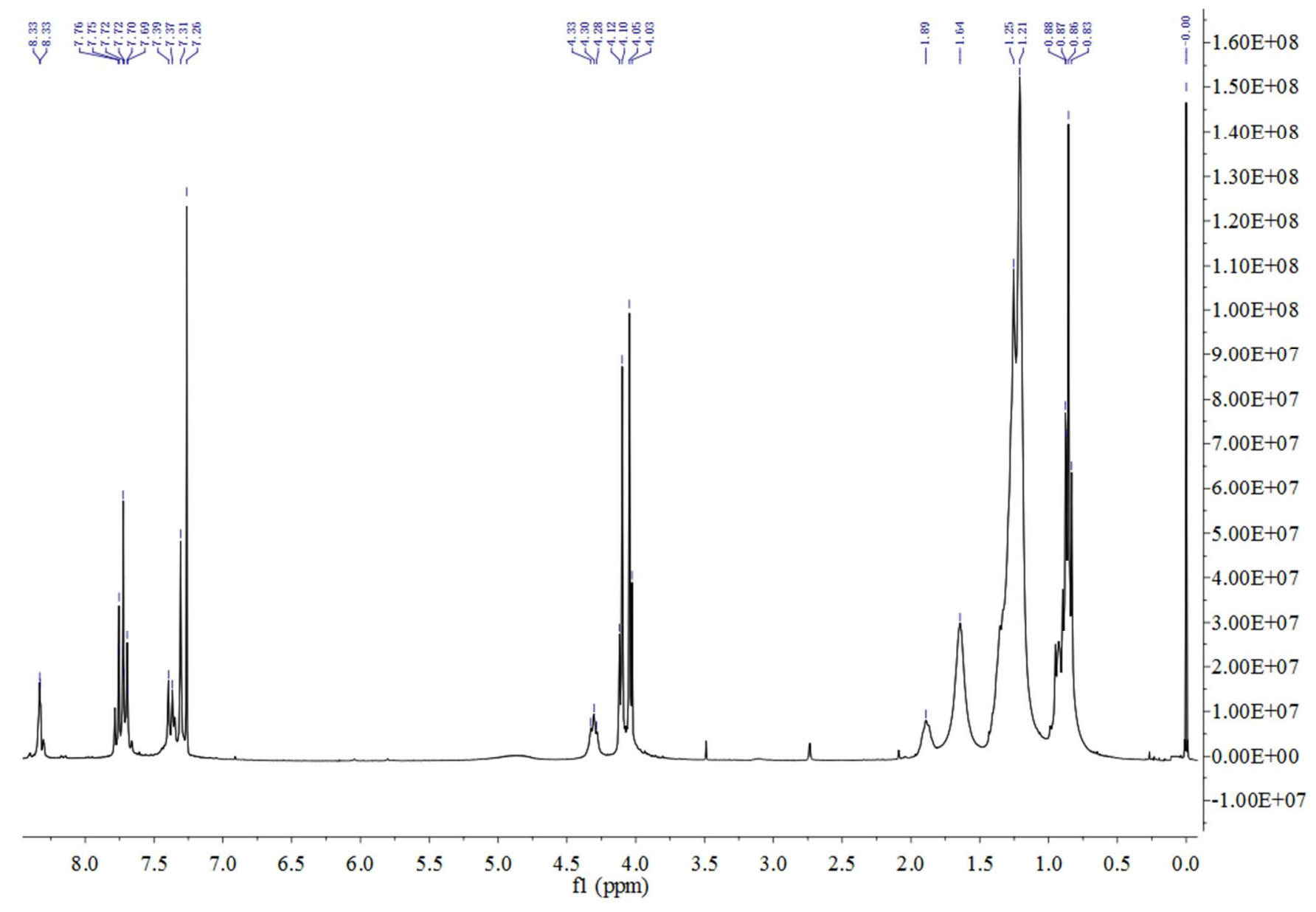

Figure S1. ${ }^{1} \mathrm{HNMR}$ of the synthesized SCP.

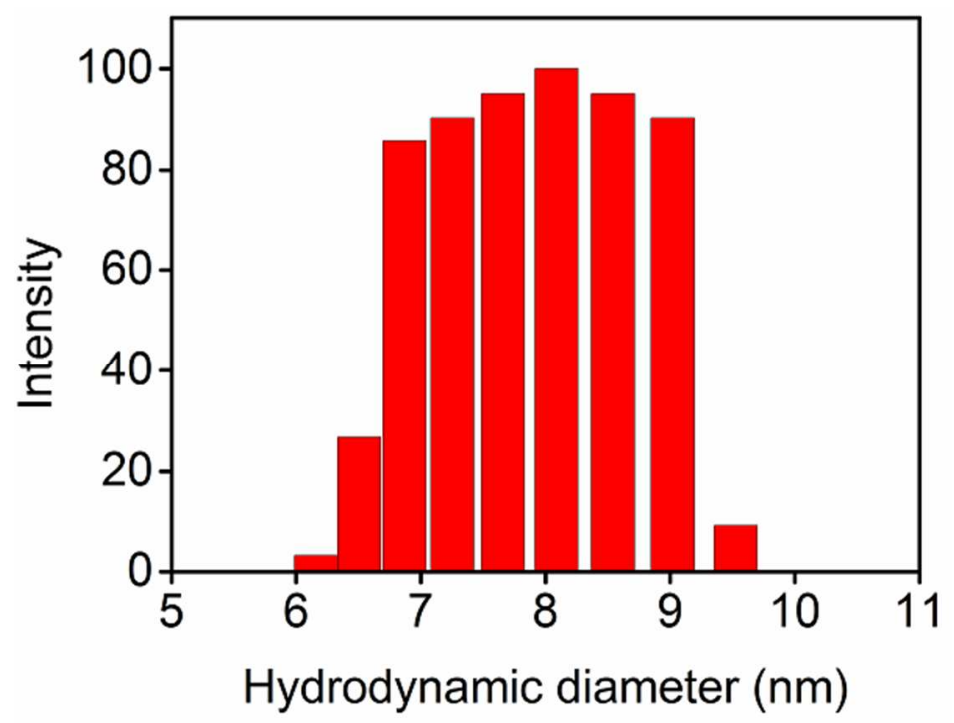

Figure S2. Hydrodynamic diameter of the SCP dots measured using DLS. 
Table S1. Electrochemical Data of SCP Dots and Calculated Energy Levels

\begin{tabular}{|c|c|c|c|c|}
\hline$E_{\text {onset }}^{\mathrm{OX}}\left(\mathrm{V}^{\mathrm{a}}\right)$ & $E_{\text {onset }}^{\mathrm{Red}}\left(\mathrm{V}^{\mathrm{a}}\right)$ & $\mathrm{HOMO}\left(\mathrm{eV}^{\mathrm{b}}\right)$ & $\mathrm{LUMO}\left(\mathrm{eV}^{\mathrm{b}}\right)$ & $E_{\mathrm{g}}\left(\mathrm{eV}^{\mathrm{b}}\right)$ \\
\hline 1.10 & -2.28 & -5.78 & -2.40 & 3.38 \\
\hline
\end{tabular}

${ }^{a}$ Potential was versus $\mathrm{Ag} / \mathrm{Ag}^{+}$. ${ }^{\mathrm{b}} \mathrm{Ferrocene}$ couple $\left(\mathrm{Fc} / \mathrm{Fc}^{+}\right)$was used as the internal reference. The energy levels were calculated using the following equations: $E_{\mathrm{HOMO}}=-\left(E_{\mathrm{Onset}}^{\mathrm{Ox}}-E_{\mathrm{Fc} / \mathrm{Fc}+}+4.8\right) \mathrm{eV}$, $E_{\mathrm{LUMO}}=-\left(E_{\mathrm{onset}}^{\mathrm{Red}}-E_{\mathrm{Fc} / \mathrm{Fc}+}+4.8\right) \mathrm{eV}, E_{\mathrm{Fc}+/ \mathrm{Fc}}=0.12 \mathrm{~V}$ vs. $\mathrm{Ag} / \mathrm{Ag}^{+}, E \mathrm{~g}=E_{\mathrm{LUMO}}-E_{\mathrm{HOMO}}$.

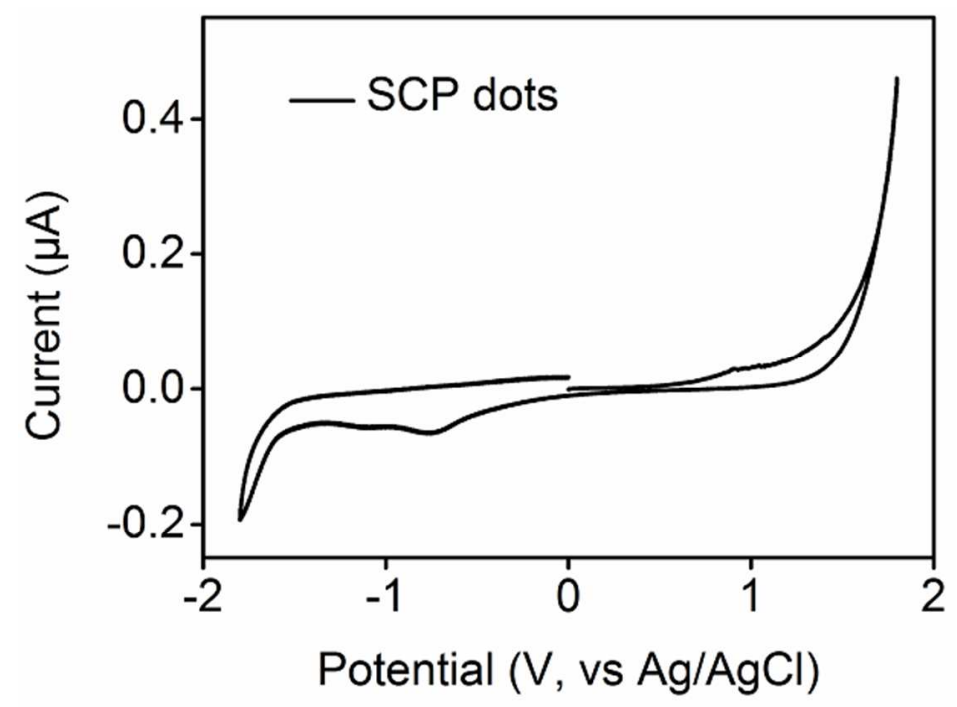

Figure S3. CV of SCP dots modified GCE in $0.1 \mathrm{M} \mathrm{pH}$ 7.4 PBS containing $0.1 \mathrm{M} \mathrm{KNO}_{3}$.

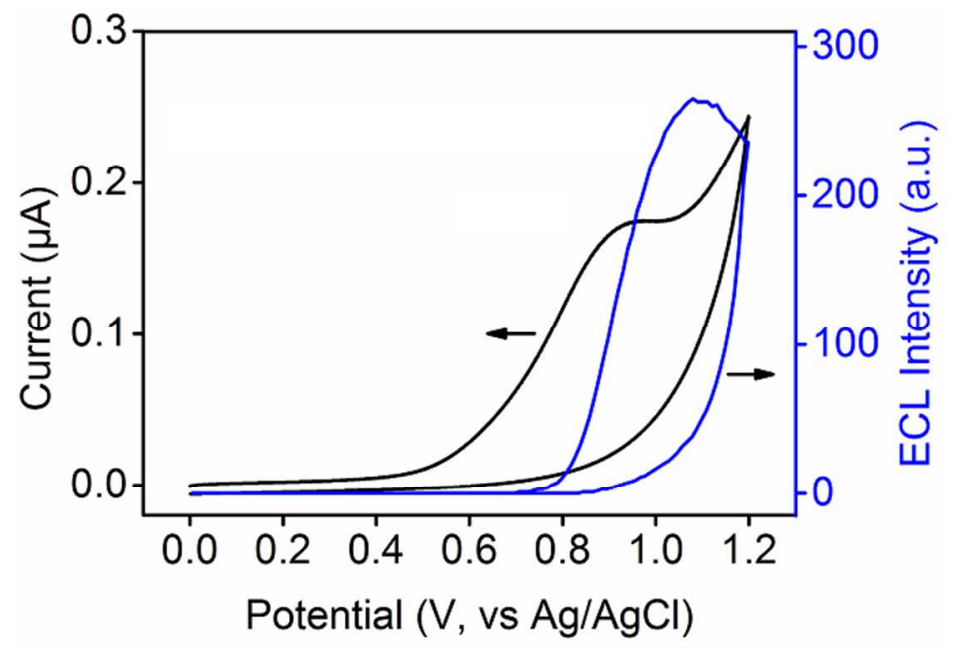

Figure S4. CV (black curve) and ECL (blue curve) of SCP modified GCE in 0.1 M pH 7.4 PBS containing $0.1 \mathrm{M} \mathrm{KNO}_{3}$ and $25 \mathrm{mM}$ TPrA as coreactant. The modified GCE was prepared by casting $20 \mu \mathrm{g} \mathrm{mL}^{-1} \mathrm{SCP}$ (in THF) and dried at room temperature. 
Table S2. Anodic ECL Behaviors of Different Luminophores

\begin{tabular}{|c|c|c|c|c|}
\hline Luminophore/coreactant & Reaction conditions & $\begin{array}{ll}\mathrm{ECL} & \text { onset } \\
\text { potential } & \end{array}$ & $\begin{array}{l}\text { ECL peak } \\
\text { potential }\end{array}$ & Ref \\
\hline SCP dot/TPrA & $0.1 \mathrm{M}$ PBS, pH 7.4 & $+0.4 \mathrm{~V}$ & $+0.78 \mathrm{~V}$ & $\begin{array}{l}\text { This } \\
\text { work }\end{array}$ \\
\hline DE-1/TPrA & $\begin{array}{l}0.1 \mathrm{M} \mathrm{Bu}_{4} \mathrm{NBF}_{4} \text { in } \\
\mathrm{MeCN}\end{array}$ & $+0.8 \mathrm{~V}$ & $+1.10 \mathrm{~V}$ & 22 \\
\hline F8BT nanoparticle/TPrA & $\begin{array}{l}0.1 \mathrm{M} \quad \mathrm{LiClO}_{4} \text { in } \\
\mathrm{MeCN}\end{array}$ & $+1.6 \mathrm{~V}$ & $+1.8 \mathrm{~V}$ & 18 \\
\hline MEH-PPV dots/TPrA & 0.1 M PBS, pH 9.0 & $+0.8 \mathrm{~V}$ & $+1.1 \mathrm{~V}$ & 25 \\
\hline PFO dots $/ \mathrm{C}_{2} \mathrm{O}_{4}{ }^{2-}$ & $0.1 \mathrm{M}$ PBS, pH 6.5 & $+0.7 \mathrm{~V}$ & $+1.9 \mathrm{~V}$ & 26 \\
\hline $\mathrm{Ru}(\mathrm{bpy})_{3}{ }^{2+} / \mathrm{TPr} \mathrm{A}$ & $0.2 \mathrm{M}$ PBS, $\mathrm{pH} 8.5$ & $+0.6 \mathrm{~V}$ & $+0.9 \mathrm{~V},+1.1 \mathrm{~V}$ & 44 \\
\hline MPA-CdTe QDs & $0.1 \mathrm{M}$ PBS, pH 9.3 & $+0.8 \mathrm{~V}$ & $+1.17 \mathrm{~V}$ & 45 \\
\hline MPA- CdTe QDs/ $/ \mathrm{SO}_{3}{ }^{2-}$ & $0.1 \mathrm{M}$ PBS, pH 7.5 & $+0.6 \mathrm{~V}$ & $+0.90 \mathrm{~V}$ & 47 \\
\hline $\begin{array}{ll}\text { dual-stabilizer-capped } & \text { CdTe } \\
\text { QDs/DBAE } & \\
\end{array}$ & $0.1 \mathrm{M}$ PBS, pH 9.2 & $+0.6 \mathrm{~V}$ & $+0.85 \mathrm{~V}$ & 48 \\
\hline
\end{tabular}
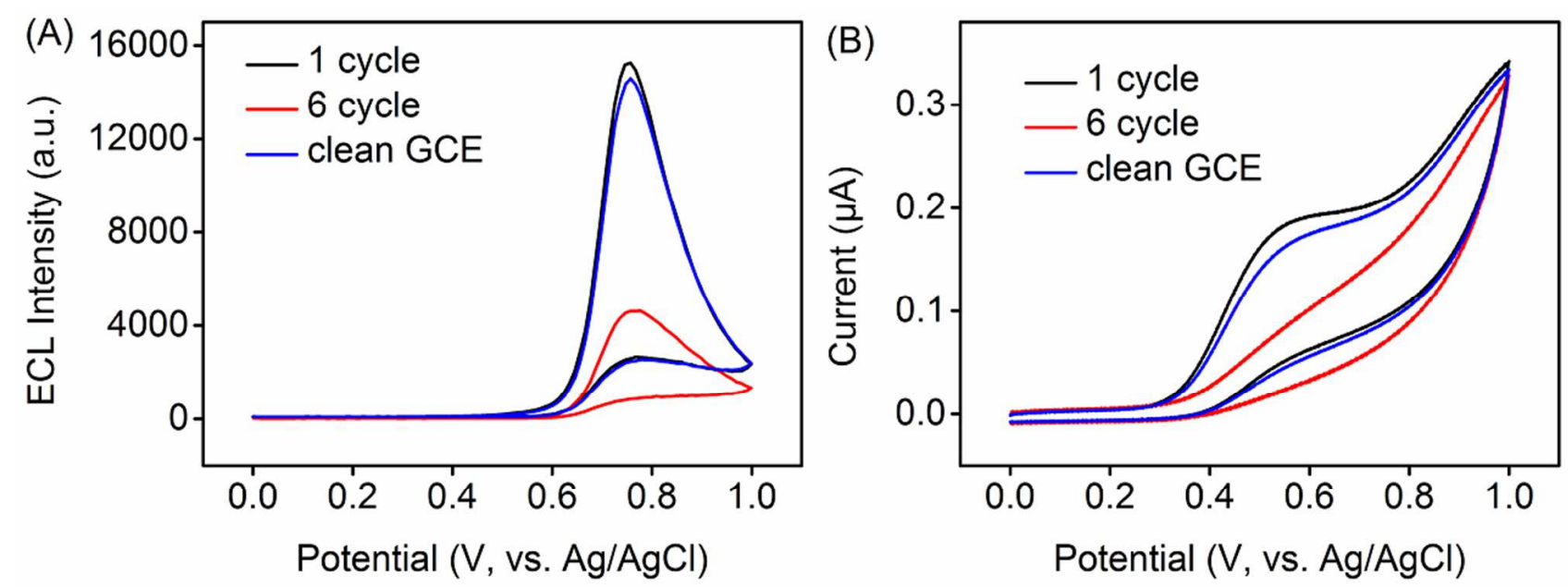

Figure S5. ECL (A) and CV (B) of SCP dots solution $\left(20 \mu \mathrm{g} \mathrm{mL}^{-1}\right)$ at GCE with $30 \mathrm{mM}$ TPrA as coreactant. 


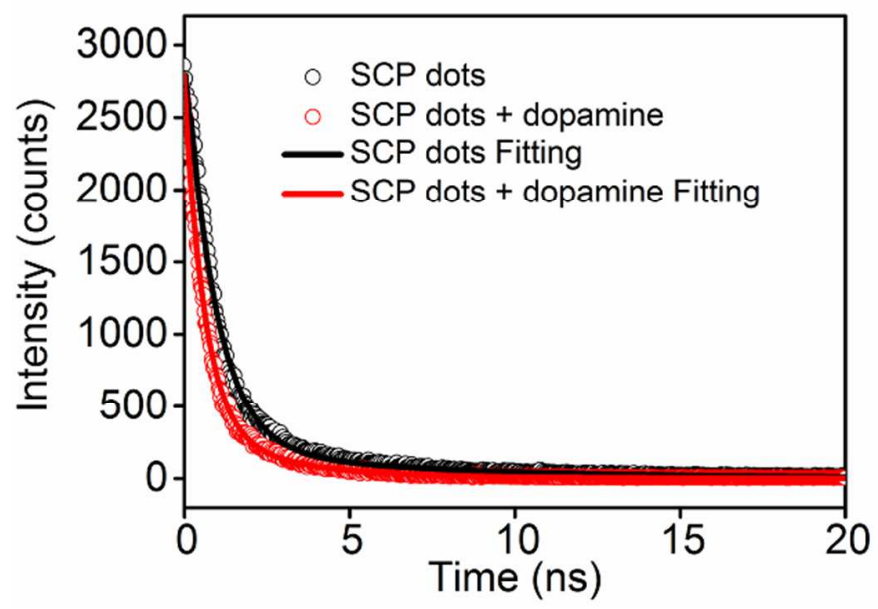

Figure S6. Time-resolved fluorescence decay of $20 \mu \mathrm{g} \mathrm{mL} \mathrm{m}^{-1} \mathrm{SCP}$ dots (experimental data, black circles; fitting, black solid line), $20 \mu \mathrm{g} \mathrm{mL}^{-1} \mathrm{SCP}$ dots after adding $10 \mu \mathrm{M}$ oxidized dopamine (experimental data, red circles; fitting, red solid line).
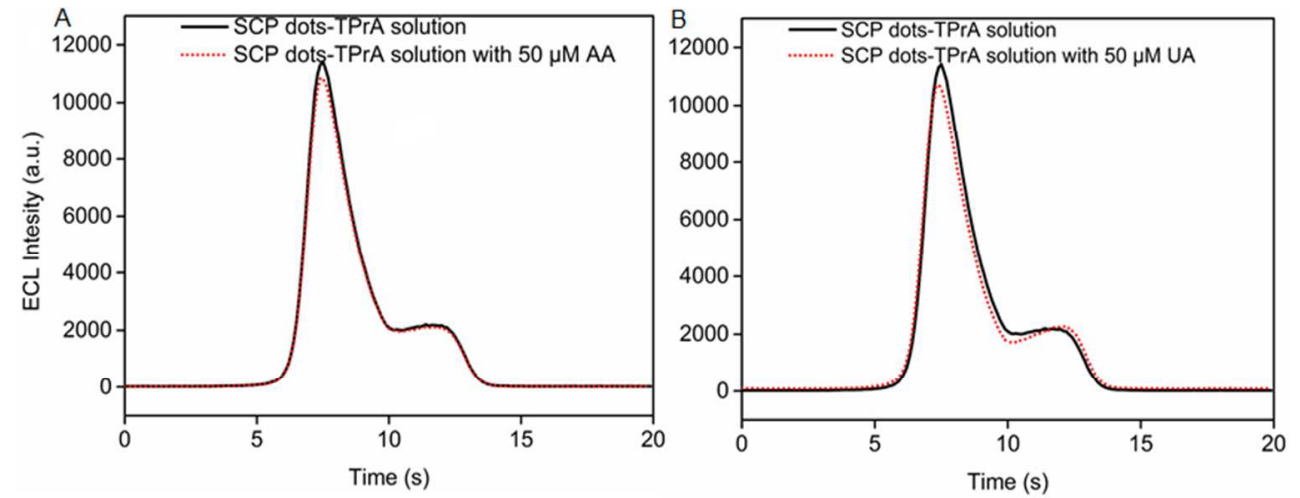

Figure S7. ECL curves of $0.1 \mathrm{M} \mathrm{pH}$ 7.4 PBS containing $20 \mu \mathrm{g} \mathrm{mL} \mathrm{L}^{-1} \mathrm{SCP}$ dots, $25 \mathrm{mM}$ TPrA and $0.1 \mathrm{M} \mathrm{KNO}_{3}$ in absence 0 (solid line) and presence of $50 \mu \mathrm{M}$ AA (A, dash line) and UA (B, dash line).

\section{References}

(1) Dai, C. H.; Wang, Y. X.; Quan, Y. W.; Chen, Q. M.; Cheng, Y. X.; Zhu, C. J. J. Polym. Sci. Pol. Chem. 2014, 52, 3080-3086.

(2) Ma, X.; Jiang, X. X.; Zhang, S. W.; Huang, X. B.; Cheng, Y. X.; Zhu, C. J. Polym. Chem. 2013, 4, 4396-4404. 